BULL. AUSTRAL. MATH. SOC.

VOL. 25 (1982), 397-403.

\title{
LINE-RANK 3 AFFINE PLANES
}

\section{Michael J, Kallaher and Graham Kelly}

\begin{abstract}
We consider finite affine planes having a collineation group acting as a rank 3 permutation group on the affine lines. By a classical result of A. Wagner, such affine planes are translation planes. We show that if, in addition, the plane has odd dimension or dimension 2 over its kernel, then the plane is Desarguesian.
\end{abstract}

\section{Introduction}

Many people have investigated automorphism groups of designs having the property that the action on the points of the design is rank 3 . See, for example, either Kallaher [2] or Lüneburg [5, Chapter III].

The authors decided to consider non-trivial $2-(v, k, \lambda)$ designs having an automorphism group that is a rank 3 group on the blocks of the design. Early in our investigation we realized that nothing was known about the special case when the design is an affine plane. By the famous Wagner theorem (Theorem 15.16 in Lüneburg [5]) the plane is a translation plane and the group contains the group of translations. This article describes what else can be said about such affine planes. We show in Section 3 that if the plane, as a translation plane, has characteristic 3 , has odd dimension over its kernel, or has dimension 2 over its kernel, then the plane is Desarguesian (Theorem 3). This leads to the conjecture that all such affine planes are Desarguesian.

Received 21 December 1981. The first author was supported in part by an ISF grant while the second author held a Postdoctoral Fellowship at Washington State University (1980-81) during the preparation of this work. 
Section 2 contains two results on finite translation planes with a collineation group doubly transitive on the line $l_{\infty}$. These results, which are needed to prove the results in section 3, are complementary to the Czerwinski-Schulz result on such translation planes (see Theorem 39.3 in Lüneburg [5]).

We assume the reader is familiar with the basic theory of affine and projective planes given in Dembowski [1] and Lüneburg [5]. We also assume that the reader is familiar with the view of translation planes or vector spaces over their kernel as given in Kallaher and Ostrom [3]. In particular, we shall use the nomenclature of that article.

\section{Preliminary results}

Let $\pi$ be a finite translation plane of dimension $d$ over its kernel $K=\mathrm{GF}(q)$, where $q=p^{k}$ with $k \geq 1$ and $p$ a prime. Furthermore, let $G$ be a collineation group of $\pi$ which is doubly transitive on the line $\tau_{\infty}$. By results of Czerwinski and Schulz (Theorem 39.3 in Lïneburg [5]), if $p=2$ or if $G$ contains no Baer involution then $\pi$ is either Desarguesian or a Lüneburg plane. In this section we investigate such planes with additional hypotheses, and two theorems are proven. The first is as follows.

THEOREM 1. Let $\pi$ be a finite translation plane of dimension $d$ over its kermel $K=\mathrm{GF}(q)$, where $q=p^{k}$ with $k \geq 1$ and $p$ a prime, and let $G$ be a collineation group of $\pi$ which is doubly transitive on the affine points of $\pi$ and doubly transitive on the points of $\tau_{\infty}$. If $d$ is odd then $\pi$ is Desarguesian.

Proof. If $p=2$ then Theorem 39.3 in Lüneburg [5] implies $\pi$ is Desarguesian or a Lüneburg plane. But $d=2$ for the Lüneburg planes. Thus the theorem holds if $p=2$. Assume $p>2$. If $d=1$ then $\pi$ is Desarguesian. Thus, assume also that $d>1$. Without loss of generality we may assume $G$ contains the group $T$ of translations of $\pi$. Then $G=T G_{0}$ where $O$ is the origin of $V$, and $G_{0}$ is doubly transitive on the points of $l_{\infty}$, since $T$ fixes $l_{\infty}$ pointwise.

Let $V$ be a point of $l_{\infty}$. Since $G_{0}$ is doubly transitive on $\tau_{\infty}$, 
the order $\left|G_{0, V}\right|$ is divisible by $q^{d}=\left|z_{\infty}-\{V\}\right|$. Also, the group $G_{V}$ is solvable by Lemma 2.13 of Kallaher and Ostrom [3]. Now the group $G_{O V}=T_{1} G_{O, V}$, where $T_{1}$ is the subgroup of translations with center $V$. From the double transitivity of $G$ on the points of $\pi$ it follows that $G_{O V}$ is doubly transitive on the affine points of $0 \mathrm{~V}$. By Theorem 37.7 of Lüneburg [5] the group $G_{O, V}$ induces on the affine points of $O V$ a subgroup of $\Gamma L\left(1, p^{k d}\right)$. (The exceptional groups cannot occur since $d$ is odd and at least 3.$)$ Now $q^{d}=p^{k d}$ does not divide the order of $\Gamma L\left(1, p^{k d}\right)$. Since the induced group is $G_{0, V} / L$, where $L$ is the order of affine perspectivities in $G_{O, V}$ with axis $O V$, it follows that $G_{O}, V$ must contain non-trivial affine elations with axis $O V$. Since $G$ is doubly transitive on $\tau_{\infty}$ and $T<G$, it follows that every affine line of $\pi$ is the axis of a non-trivial affine elation (shear). By Theorem 39.2 of Lüneburg [5] the plane $\pi$ is Desarguesian.

The last theorem of this section considers translation planes of dimension 2 .

THEOREM 2. Let $\pi$ be a finite translation plane of dimension $d$ over its kermel $K=\mathrm{GF}(q)$, where $q=p^{k}$ with $k \geq 1$ and $p$ a prime, and let $G$ be a collineation group of $\pi$ which is doubly transitive on a line $l_{\infty}$. If $d=2$ then $\pi$ is either Desarguesian or a Lüneburg plane.

Proof. If $p=2$ this theorem follows from Theorem 39.3 in Lüneburg [5]. Assume $p>2$. If $T$ is the group of translations of $\pi$, then the group $H=T G$ is also doubly transitive on $\tau_{\infty}$. Since $T$ fixes the line $l_{\infty}$ pointwise, the subgroup $H_{0}$, where 0 is the origin of $\pi$, is doubly transitive on $l_{\infty}$.

Let $V$ be a point of $z_{\infty}$, and consider $H_{1}=H_{0, V}$. Since $H_{0}$ is doubly transitive on $l$, the order $\left|H_{1}\right|$ is divisibly by $q^{2}$. On the other hand the group $H_{1}$ induces on the affine line $O V$ a group $\bar{H}_{1}$ of semi-linear transformations considering $O V$ as a vector space of 
dimension 2 over $K=\mathrm{GF}(q)$ (see Lemma 2.11 in Kallaher and Ostrom [3]). Thus, $\bar{H}_{1} \leq L(2, q)$. Since $q^{2}$ does not divide $|\Gamma L(2, q)|=k q\left(q^{2}-1\right)(q-1)$, as in the last proof $H$ contains non-trivial affine elation, and thus Theorem 39.2 of Lüneburg [5] implies $\pi$ is Desarguesian.

The Czerwinski-Schulz theorem does apply to translation planes of nonsquare order since such planes cannot have Baer involutions. However, it does not apply to translation planes of odd dimension over their kernel, for such planes do have Baer involutions.

\section{Line-rank 3 affine planes}

In this section we prove the main result of this article. To facilitate the discussion we make the following definition.

DEFINITION. A line-rank 3 affine plane is a finite affine plane $\pi$ with a collineation group $G$ which is a rank 3 permutation group on the affine lines of $\pi$. The group $G$ is called a line-rank 3 collineation group.

The following is an immediate consequence of the famous Wagner theorem on affine line transitive collineation groups.

LEMMA 1. A Zine-rank 3 affine plane $\pi$ is a translation plane and a line-rank 3 collineation group $G$ of $\pi$ contains the group $T$ of transiations of $\pi$.

Proof. The line-rank 3 collineation group $G$ of $\pi$ is transitive on the affine lines of $\pi$. By Wagner's theorem (Theorem 15.16 of Lüneburg [5]) the plane $\pi$ is a translation plane and $T<G$.

Let $\pi$ be a line-rank 3 affine plane with line-rank 3 collineation group $G$. Then $\pi$ is a finite translation plane. Let $K=G F(q)$, where $q=p^{k}$ with $k \geq 1$ and $p$ a prime, be the kernel of $\pi$ and let $d$ be the dimension of $\pi$ over $K$ (see Section 2 of Kallaher and Ostrom [3]). Thus $\pi$ has order $q^{d}$, and $\pi$ is a vector snace of dimension $2 d$ over $K$. If $T$ is the group of translations of $\pi$ then

$$
G=I G_{0}
$$


where 0 is the origin (zero vector) of $\pi$. Furthermore, the group $G_{0}$ is a group of semi-linear transformations over $K$ on the vector space $\pi$.

Since $G$ is transitive on the affine lines of $\pi$, the group $G$ is transitive on the points of $\tau_{\infty}$, the line at infinite of $\pi$. Consider an affine line $l$ and the subgroup $G_{l}$. The rank 3 property of $G$ implies that $G_{l}$ has exactly three orbits in the set of affine lines of $\pi$. Since $G_{l}$ fixes the point $V=l \cap l_{\infty}$ on the line $l_{\infty}$ these three orbits must be $\Lambda_{1}=\{l\}$, the set $\Lambda_{2}$ consisting of all affine lines in $\pi$ meeting $z_{\infty}$ in the point $V$, and the set $\Lambda_{3}$ consisting of all affine lines in $\pi$ not meeting $l_{\infty}$ at the point $V$. It follows that $G_{l}$ is transitive on the points of the set $Z_{\infty}-\{V\}$. Because $G_{Z} \leq G_{V}$ we have proven the following:

LEMMA 2. Let $\pi$ be a line-rank 3 affine plane with a line-rank 3 collineation group $G$. The group $G$ is doubly transitive on $\tau_{\infty}$, the line at infinity.

Since $T<G$ the group $G$ is transitive on the affine points of $\pi$. Consider the origin 0 and a line $z$ through 0 . If $U=l \cap l_{\infty}$ and $T_{2}$ is the group of all translations with center $U$, then

$$
G_{2}=T_{2} G_{0,2}
$$

since $T_{2}$ fixes $l$ and is transitive on the affine points of $l$. The rank 3 property of $G$ implies that $G_{Z}$ is transitive on the set of affine lines $m$ with $m \cap Z_{\infty}=U$ and $m \neq Z$.

Because $T$ fixes every point of $l_{\infty}$, equation (1) above implies the group $G_{0}$ is doubly transitive on the points of $Z_{\infty}$. It follows that $G_{0}$ has two orbits on the set of affine lines of $\pi ;$ namely, the set $\Sigma_{1}$ consisting of all lines through 0 and the set of $\Sigma_{2}$ consisting of all remaining affine lines of $\pi$. Thus, on the set of affine points the group $G_{0}$ has at most two orbits (see 2.3.1 in Dembowski [1]). Since $\{0\}$ is one such orbit, the group $G_{0}$ has a second orbit consisting of all the 
remaining affine points. We have proven the following lemma.

LEMMA 3. Let $\pi$ be a line-rank 3 affine plane with a line-rank 3 collineation group $G$. The group $G$ is doubly transitive on the affine points of $\pi$.

One consequence of Lemma 3 is the fact that a Lïneburg plane cannot be a line-rank 3 affine plane. For the full collineation group of a Lüneburg plane is a rank 3 permutation group on the affine points of the plane.

We can now prove the main result of this article.

THEOREM 3. If $\pi$ is a line-rank 3 affine plane with a line-rank 3 collineation group $G$, then $\pi$ is a translation plane and $G$ contains the group $T$ of transzations. Furthermore, the plane $\pi$ is Desarguesian if any one of the following conditions holds:

(i) the characteristic $p$ of $\pi$ is 2 ;

(ii) the dimension $d$ of $\pi$ over its kermel $K=\operatorname{GF}(q)$ is odd;

(iii) the dimension $d$ of $\pi$ over its kemel $K=G F(q)$ is 2 .

Proof. The first statement is just Lemma 1. The second statement follows from Theorem 39.3 of Lüneburg [5], Lemmas 2 and 3, Theorems 1 and 2 , and the remark after Lemma 3 .

\section{References}

[1] P. Dembowski, Finite geometries (Ergebnisse der Mathematik und ihrer Grenzgebiete, 44. Springer-Verlag, Berlin, Heidelberg, New York, 1968).

[2] Michael J. Kallaher, "A survey of weak rank 3 affine planes", Proc. Internat. Conf. Projective Planes, 121-143 (Washington State University, Pullman, Washington, 1973. Washington State University Press, Pullman, Washington, 1973). 
[3] M.J. Kallaher and T.G. Ostrom, "Collineation groups irreducible on the components of a translation plane", Geom. Dedicata 9 (1980), $153-194$.

[4] Marion E. Kimberley, "On collineations of Hadamard designs", J. London Math. Soc. (2) $6(1972 / 3), 713-724$.

[5] Heinz Lüneburg, Translation planes (Springer-Verlag, Berlin, Heidelberg, New York, 1980).

Department of Mathematics,

Washington State University,

Pul Iman,

Washington 98164 ,

USA;

AMP Society,

GPO Box 4134 ,

Sydney,

New South Wales 2001 ,

Australia. 